\title{
Fungal Future: A review of mycelium biocomposites as an ecological alternative insulation material
}

\author{
Owen Robertson ${ }^{1}$, Frederik Hogdal ${ }^{1}$, Lukas Mckay ${ }^{1}$, Torben Lenau1 \\ ${ }^{1}$ Technical University of Denmark \\ owen.i.robertson@gmail.com \\ frhog@dtu.dk \\ lukasmckay@gmail.com \\ lenau@mek.dtu.dk
}

\begin{abstract}
Reducing the use of non-renewable resources is a key strategy for transition to circular economy. Mycelium is the vegetative part of fungus which can cement particulate substrate and can be formed into any shape if grown in a mould. Mycelium biocomposites (MBs) are rapidly being seen as green alternative for many hydrocarbon-based products, including Expanded Polystyrene (EPS) used for insulation in the construction industry. This is largely due to its comparable acoustic/insulative properties, superior fire safety and minimal environmental impact. Furthermore, as MBs can utilize low cost readily available commercial waste products such as wheat husks as a composite substrate, a clear value chain upscaling can be envisioned.

Throughout its linear lifecycle, EPS insulation pose numerus environmental issues, including high resource use and challenges in its end of life disposal. Even if disposed correctly it can take thousands of years to degrade, evidently making it extremely difficult to properly contain. This has resulted in bioaccumulation of toxic chemicals in food webs across the planet.

Conversely, MBs are biodegradable and importantly can be used as raw material for the production of more MBs. When comparing life cycle assessment (LCA) and production, MBs are estimated to hold clear advantages in terms of reduced $\mathrm{CO} 2$ out put and costs. It is thus clear it holds the potential to become an ideal candidate for a "cradle to cradle" economy, in this sector.

Despite these attributes, MB insulation still have evident disadvantages when compared to their hydrocarbon counterparts and could hinder its adoption on a commercial scale. These include higher density and issues with water uptake. Furthermore, there can be wide variability in material performance on the basis of which substrate composition fungal strain, incubation conditions and manufacturing techniques are used. This coupled with the relatively sparse research in this field makes full assessments and comparisons between studies more difficult. New design approaches will also have to be considered when producing MB due to the additional factors of working with growing organisms. MB offers new degrees of freedom to the designer to create shapes and internal geometries yet only seen when using 3d-printing but at a price which potentially is suited for mass production low price products such as advanced
\end{abstract}


packaging products and composite sandwich structures with tailor-made porous internal stiffening elements.

Fully commercialised MB products have however begun to emerge. These still largely rely on costly labour-intensive manufacturing practices but present as an ideal target for optimization through factory atomisation. Integrating other emerging technologies such as $3 \mathrm{D}$ printing and computerised optimizations could also allow for controlled growth of internal structures of MBs. This would enable the creation of novel green materials with greatly improved performance characteristics which can be tailors to specific requirements. Ultimately furthering their viability as a green option to traditional EPS insulation.

\section{Keywords: Mycelium biocomposties (MBs), Insulation, Expanded Polystyrene (EPS), Life cycle asssment (LCA), Circular economy}

\section{Introduction}

The construction sector in Europe accounts for about half of all our extracted materials and energy consumption and roughly a third of our water consumption and waste generation (European Commission., 2010). A green material revolution is clearly required in order to address the huge "cradle to the grave" environmental issues with current methods.

A prime candidate that could be targeted are polymeric foams, such as EPS. They are commonly used for lightweight fill and thermal insulation and housing construction. These petroleum-based materials have numerous positive attributes such as being hydrophobic, lightweight and having excellent sound/thermal insulative properties (Yang et al., 2017).

However, as with most man-made materials, there are often huge ecological and societal negative impacts throughout their associated lifecycle. From the drilling of oil to eventual disposal, these hydrocarbon materials are non-renewable, in addition to their production requiring complex manufacturing processes, massive energy inputs, and associated waste streams (Bandyopadhyay \& Basak, 2007). Furthermore, they can take thousands of years to decompose, posing substantial problems with regards to reuse recycling and landfill operation (Yang et al., 2017). Large quantities of polymeric foams have thus inevitably found their way into the wider terrestrial and marine environment. Significantly, these plastics have an affinity for chemical pollutants such as heavy metals and carcinogenic chemicals, resulting in their bioaccumulation into food webs (Rochman et al., 2013).

Over the last decade, rapid advances in MB technology have highlighted their potential as a green alternative to many materials used in manufacturing today, such as building insulation or packaging and are already seeing the emergence of their commercial use (fig.1) (Yang et al., 2017; Jones et al., 2020; Ecovative, n.d., 2020; Gryphon, n.d., 2020). Mycelium is a fastgrowing vegetative part of mushrooms which is a safe, inert, renewable, natural and green material. This has the ability to rapidly produce miles of self-assembling tiny fibres named hypha which can envelop, digest and bind surrounding material into a strong and biodegradable material, which can be part of a circular economy (fig. 2) (Schiffman, 2013).

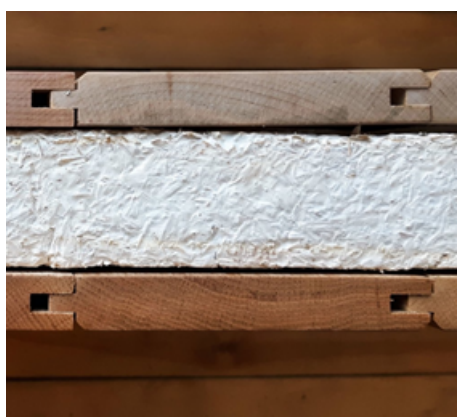


Figure 1. Mycelium composites as insulating cores in Gryphon doors.

A typical cycle time is about 5-8 days, to adequately colonize the reinforcement fibres and/or core material, and longer colonization leads to stiffer and stronger final materials (Jiang et al., 2013).

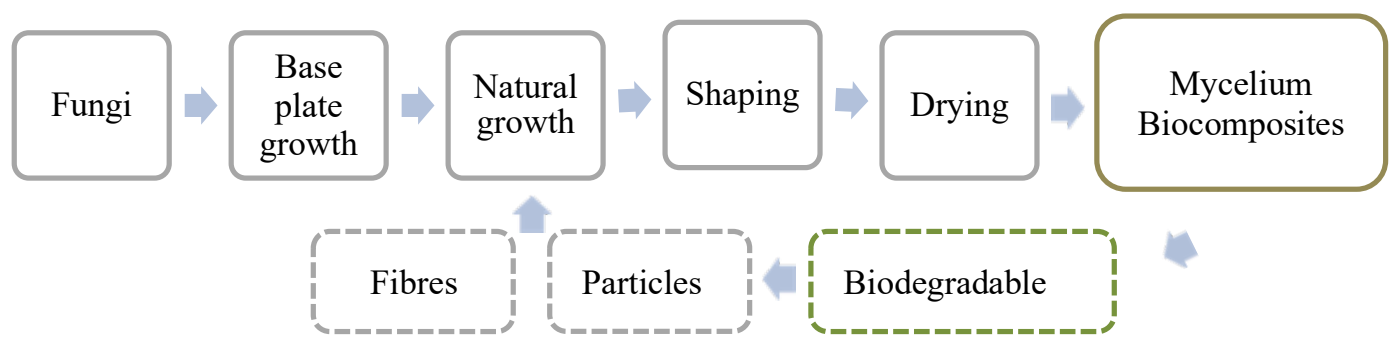

Figure 2. Lifecycle of MBs, highlighting potential circular economy input.

Due to the vast number of fungal strains, growth mediums and environmental factors that can influence the growth characteristics of the mycelium, numerous studies have demonstrated the capability of this material's wide range of insulation and mechanical properties that are comparable to conventional plastic foams or even soft woods; heat pressing shifted mycelium composites from foam-like performance to cork and wood-like performance (Appels et al., 2019). If grown in a mould, any desired shape can be produced and used as a direct replacement to conventional materials (Abhijith et al., 2018). Furthermore, MBs have also been demonstrated to process intriguing properties in regards in both thermal and acoustic insulation.

Despite having many of the required comparable performance characteristics for in many of the required attributes of polymeric foams (Yang et al., 2017; Jones et al., 2020), there remains several obstacles to overcome before it can match or surpass conventional insulators. These include high moisture uptake and lower dry density (Jones et al., 2020). In addition, it has been observed that the hyphal density in the core of these MBs was significantly lower than at the surface, with negligible differences observed as a function of growth periods. This can be attributed to the reduced oxygen diffusion into the bulk of the composite, an essential ingredient for mycelial growth (Jones et al., 2018). This could thus constrain its practical applications due to the limit of material thickness capable of being manufactured.

As mycelium structures are grown, rather than manually assembled, it is however conceivable if provided with a growth scaffold such as those utilized in 3D printed tissue fabrication (Shafiee \& Atala, 2016), the creation of predetermined and complex internal structures can be achieved. Furthermore, these will also allow for the control of environmental growth conditions throughout the material, offering a unique opportunity to create MBs with previously unobtainable performance properties not limited to insulation. This concept could 
be further refined through biomimetic design as well as the utilizing computerised topography optimisation to create structures. Allowing for the creation of ecological insulators with unsurpassed performance characteristics.

\section{Thermal and acoustic insulation properties of MBs}

Lower thermal conductivity is associated with better insulation materials and is primarily influenced by the density of material (Uysal et al., 2004). The strong relationship between thermal conductivity and material density can be attributed to the extremely low thermal conductivity of dry air $(26.2 \times 10-3 \mathrm{~W} / \mathrm{m} \cdot \mathrm{K}$ at $0.1 \mathrm{MPa}, 300 \mathrm{~K})$ (Kadoya et al., 1985).

This is also apparent with MBs when high performing natural insulators such as hemp or straw are incorporated as a growth substrate, both demonstrating low thermal conductivity $(0.039$ $0.08 \mathrm{~W} / \mathrm{m} \cdot \mathrm{K}$ ) and density (57-99 kg/m3) (Gryphon., 2020; Elsacker et al., 2019). These values make them within the competitive range in terms of thermal conductivity when compared to traditional EPS insulation (0.03-0.04 W/mK). However these man made materials are still significantly lighter $(16-48 \mathrm{~kg} / \mathrm{m} 3)$ (Papadopoulos, 2005). Furthermore the mechanical properties of such mycelium materials were suboptimal when again compared to EPS (Elsacker et al., 2019). Conversely, Yang et al, (2017) demonstrated a mycelium biofoam which met or exceeded characteristics of the conventional EPS foams except density. This was achieved using a substrate mix combination of various materials, including wood pulp, wheat bran, millet grain, natural fibre, and calcium sulphate. These findings thus imply that there is huge potential to further develop and optimize such materials to further increase their competitiveness as a viable alternative.

Besides thermal insulation, MBs have shown huge potential for acoustic insulation. Acoustic absorbers convert the mechanical motion of air molecules travelling in sound waves into heat energy, reducing reflected noise strength and sound accumulation (Bell, 2017).

Pelletier et al, (2013) tested MB panels using a range of natural substrates and reported an acoustic absorption of over $70-75 \%$ at $1000 \mathrm{~Hz}$ (the dominant frequencies from road traffic). Comparisons among audio spectra show the highest absorption occurred with a growth substrate composed of $50-50 \%$ switchgrass-sorghum.

Not only the composition of the material but also physical structure plays a significant role in sound dampening. These can be designed to alter phase shifts and destructive interference for specific frequencies, drastically reducing reflected sounds (Xu \& Huang, 2018). It is conceivable that future panels could be designed with both thermal and acoustic insulation considerations and could be tailored for their required characteristics depending on habitat.

\section{Fire safety properties of MBs}

Although relatively few studies have investigated the behaviour of mycelium composites towards fire and pyrolysis, there is increasing evidence for their effectiveness regarding fire 
safety (Jones et al., 2020). Mycelium by itself lacks notable fire-retardant characteristics. However, MBs can obtain these by incorporating fillers or substrates that are rich in naturally occurring/synthetically produced silica ( $\mathrm{SiO} 2)$ and natural phenolic polymers, such as lignin. These have demonstrated greatly improved fire reaction, thermal degradation and safety properties (Jones et al., 2018). Significantly, rice hulls have been found to contain $15-20 \mathrm{wt} \%$ silica and $25-30 \mathrm{wt} \%$ lignin (Ismail \& Waliuddin, 1996). Glass fines (silica) and rice husks are both considered industrial waste products and are readily available at low value (Jones et al., 2018; Oecd et al., 2009).

These mycelium composites had significantly lower average peak heat release rate and a longer estimated time to flashover than the synthetic insulation materials considered (Jones et al., 2018). Flashover is the near simultaneous ignition of all exposed materials in an enclosed area and is a regular and extremely dangerous occurrence in building fires, furthermore fires that reach flashover are around ten times more dangerous than fires that do not (Liang et al., 2013).

Plastic foams are often major contributors to fires involving rapid flame spread which generate high volumes of smoke and toxic gases including carbon monoxide $(\mathrm{CO})$ and hydrogen cyanide

(Stec \& Hull, 2011; Rossi et al., 2001). Regardless of the dangers associated with flashover and heat release, the majority of fire related deaths are a result of these gases rather than burns, trauma or other causes (Babrauskas et al., 1992). CO exposure results in incapacitation and death at extremely low concentrations ( $1500 \mathrm{ppm}$ can cause death within an hour) and is considered the largest individual hazard (Neviaser \& Gann, 2004). Mycelium composites containing rice husks or synthetic silica release significantly less $\mathrm{CO} 2$ and smoke when combusted, compared to hydrocarbon insulators (Jones et al., 2018; Jones et al., 2020).

\section{Water absorption properties of MBs}

Experiments on MBs have shown they typically have an affinity towards water rapid absorption, increasing in weight by $\sim 40-580 \mathrm{wt} \%$ when in contact with water for $48-192 \mathrm{~h}$ (Nava et al., 2016; Appels et al., 2019). The fastest weight increase occurred within the first $3 \mathrm{~h}$, with an increase of $\sim 220 \mathrm{wt} \%$ for both cotton bur fibre and rapeseed straw based composites (Appels et al., 2019).

This is perhaps the largest issue preventing their wider adoption into a commercial market. However, as acoustic or thermal insulation is typically used in internally dry locations this may arguably not be a significant problem.

There is also mounting evidence demonstrating methods to limit water uptake. Using particulate growth substrates such as beech sawdust, showed a reduced susceptibility, increasing its weight of $23 \mathrm{wt} \%$ over $3 \mathrm{~h}$ contact with water, which slowly increases to $43 \mathrm{wt} \%$ over 192h (Appels et al., 2019). This is likely due to the smaller void content and higher material density present in composites made from fine particulate substrates (Jones et al., 2020). In addition, Beech sawdust contains $26 \mathrm{wt} \%$ hydrophobic lignin as well as its $48 \mathrm{wt} \%$ cellulose, contributing to this observation (Miklečić \& Jirouš., 2016).

The manufacturing process of mycelium composites has been shown to play a role in water uptake. Hot or cold pressed composites experienced significantly reduced 
uptake of their air-dried counterparts ( $\sim 250 \mathrm{wt} \%$ compared to $\sim 580 \mathrm{wt} \%$ ) (Jones et al., 2020) (Appels et al., 2019). This is probably due to pressed materials having lower void volumes impeding capillary action and hence reducing water uptake (Yu et al., 2007). Appels et al, (2019) also noted that the mycelium strain used can have an effect on the composites interaction with water. Species such as $T$. versicolor can form a thick hydrophobic fungal skin at the substrate air interface and saw reduced water uptake in its composites.

\section{Comparative life cycle assessment (LCA) of MBs and traditional insulators}

Two of the most important factors in a circular economy perspective are renewable feedstocks and biodegradability. MBs, as opposed to non-renewable petrochemical based polymers, will degrade in a matter of months if composted (Appels, 2020). Alternatively, the Mycelium-based products can be used as: feedstock for animals, energy production (which will affect an LCA positively), compost or re-utilized into new mycelium products (Grimm et al., 2018). The potential of circularity is underlined by the official C2C certification (Product Scorecard, n.d.). Apart from circularity, LCA's are often used as the benchmark for sustainability (Nissinen et al., 2017). Ecovative, claims that about $56 \%$ of its CO2 emissions come from the drying process of their mycelium products (Ecovative, n.d., 2020).

Based off Leiva et al, (2015) it is estimated that the production of Agaricus Bisporus (a species of mushroom) is approximately the $12.9 \%$ energy production the original blend costs ecovative. Assuming this, a comparative analysis shows EPS produces roughly 3 times the amount $\mathrm{CO} 2(6.98 \mathrm{CO} 2 / \mathrm{kg})$ than $\mathrm{MBs}(2.2 \mathrm{CO} 2 / \mathrm{kg})$ during manufacturing (Chandra et al., 2016).

The species of fungi could also to be important to an LCA, as the fungi itself respires, producing $\mathrm{CO} 2$. Currently, only a few fungi of the basidiomycetes species have been studied, however a wide range in respiration rates has been observed (Appels, 2020). Although these values are relatively small, this input on larger scale production have yet to be investigated and needs to be considered in its LCA.

\section{Comparative production costs}

MBs have also been shown to be competitive in terms of production costs when compared to EPS. Jones et al., (2018) showed volume specific costs were 6 to 12 times cheaper (AU\$40/m3 and AU $\$ 81 / \mathrm{m} 3$ respectively) than EPS (XPS, AU $\$ 491 / \mathrm{m} 3$ ). Agricultural waste used as composite raw materials for the mycelium is also much cheaper when compared to the wholesale price of polystyrene (2.1-2.3 \$US/kg) and polyurethane (8.2-10.4 \$US/kg) (Jones et al., 2020).

Significantly, roughly $90 \%$ of the total production price of one-kilogram MB is predicted to be labour associated costs. In Denmark five minutes pay equates to around DKK 19, which is more than the total cost of materials for a kilogram of mycelium material. It must however be noted, data on predicting production is still somewhat sparse lacking real-world industrial scale examples. 
The gross energy required to produce $1 \mathrm{~kg}$ of polystyrene is 90MJ (Boustead et al., 1993). Although analysis on this has not been done in regards to mycelium, the energy required is likely to be considerably lower, as none is required during mycelium's 5-7 growth period (Jiang et al., 2016).

It is clear there is considerable opportunity for optimization, especially regarding labour costs. As this manufacturing process is largely segmented and linear, automation as with many other industries likely could provide the solution to minimize costs while increasing consistency of the final product (Smunt \& Meredith, 2000).

\section{Design implications in future MBs}

Insulative properties, similar to mechanical properties, are influenced by the geometrical configuration of composite mediums, typically convection can be reduced by having small selfcontained air spaces (Springer \& Tsai, 1967). However, as more air cavities are introduced, typically the structural integrity of the material decreases and vice versa (Lorente \& Bejan, 2002). This inverse correlation dilemma can somewhat be resolved as shown by in a study by Xie et al, (2014) which highlighted a method for the structural optimization for an insulating wall combining heat flow.

To date, there are however no methods available to allow for precise or even randomized control of moulded mycelium composite internal structures. If this be achieved it would undoubtedly open to the door creation of novel mycelium based materials with a range of superior performance characteristics, which could be tailored to suit desired weight, impact absorption, thermal and moisture properties.

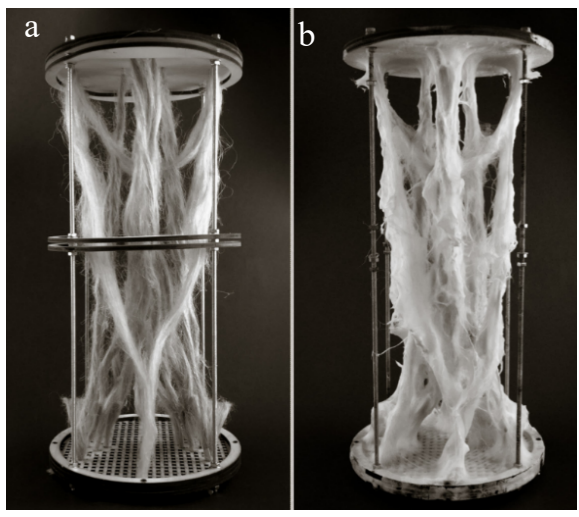

Figure 3. a- hemp fibres arranged in frame. bgrowth of mycelial onto original hemp framework.

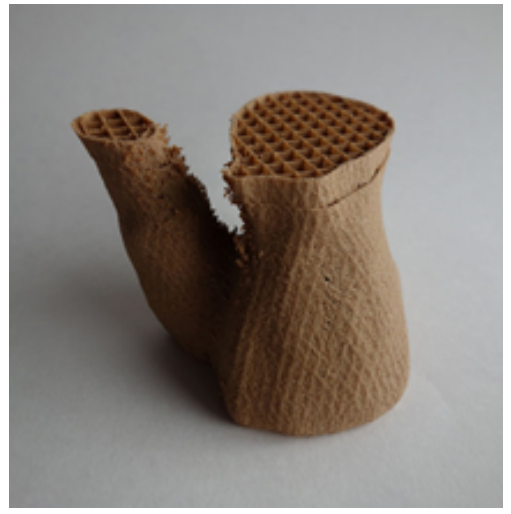

Figure 4. 3d printed mycelium inoculated object utilizing a straw based print media.

This presents a cross-domain challenge requiring both design and biological perspectives to be considered. Unlike traditional manufacturing which harvests raw material then transforms it into other products, MBs growth is an intergyral part of production and all factors influencing this must accounted for in the initial design conception. 
This added layer of complexity could however offer new design opportunities and tools, allowing for the creation previously unobtainable organic forms. The initial feasibility of this
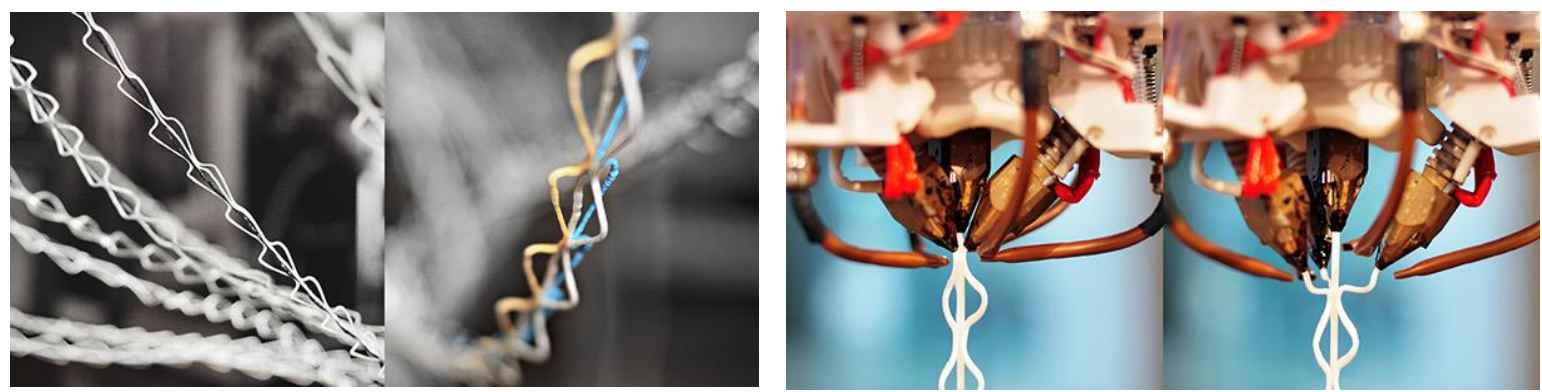

Figure 5. New spider web inspired 3D-printing of free-standing structures could make possible much faster production and allow the integration of other materials such as straw and other agricultural waste products.

concept has been highlighted in work by Tabellini, (2015) (fig.3), who used hemp fibre frames to demonstrate how mycelium growth can be influenced and guided. However, this method requires an initial supporting structure not making it a practical solution. A viable alternative is to construct celluloses rich growth scaffolds using additive manufacturing (AM - also referred to as 3d-printing). This technology can produce complex internal structures, but in general it is difficult to place material where there is no underlying support. The problem can be solved by several techniques including the addition of support structures that use liquid photo polymers or powder bed AM. As these additional steps typically increase fabrication and processing time, it has so far proven to be a significant issue preventing AM being widely adopted as an economically viable mass production method (Sasson et al,. MBs have already shown compatibility with this technology, allowing for the creation of previously unobtainable morphologies compared to moulding processes. These utilized a printable mycelium inoculated straw based or wood/paper pulp media (Goidea et al., 2020; Fairs, 2013) (fig. 4). However, this technique is still subject to the time limitations discussed. A solution could be to use a newly developed multi-extrusion 3d-print inspired by the silk spinning techniques of silkworms and spiders (The Voice of 3D Printing., 2015) (fig.5).

that would allow fibrous free standing horizontal structures without the need for extra support material, effectively eliminating the major drawbacks for both moulding/printing methods whilst allowing gas exchange deep within the structure. Combining with computerized topology optimization techniques such as those used to develop aircraft wings (Aage et al., 2017) (fig.5) will also allow for refined control of printing support structures from everything to maximal stiffness to weight ratio or thermal/acoustic properties. Such structures are today produced using 3D-printing but production times prohibit the application of 3D-printed parts

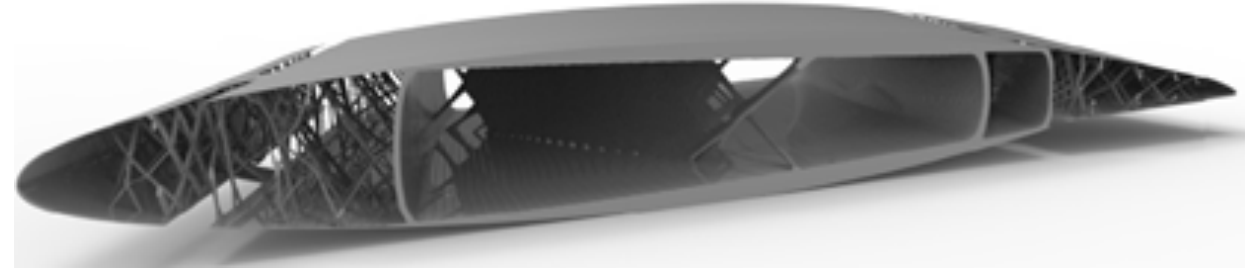

Figure 5. Cross section of computational topography optimized aircraft wing.

to high-value items such as aircraft wings. MB has the potential to make similar structures but at mass production commodity price levels. To utilise this opportunity, designers need to carry 
out their work differently while considering the internal layout of porous internal structures in the materials. To promote the application of MB in volume products, designers need access to dedicated computer support that helps in proposing topology optimized structures while at the same time considering the opportunities and limitation in the 3D-printing of MB growth scaffolds.

\section{Discussion}

There is mounting evidence that they can potentially exhibit far better fire safety parameters in addition to having comparable mechanical and insulative acoustic/thermal performance characteristics if compared to their hydrocarbon counterpart. Higher material density and water uptake could still pose a challenge to wider commercial adoption, however some progress has been made to resolve these issues. Nevertheless, literature providing a satisfactory material characterization on all these properties is still scarce as well as evident complications when comparing even similar studies. This is largely due to the wide range of parameters such as mycelium strain, growth time growth environment, growth substrate and manufacturing technique can drastically alter the material performance characteristics. It is thus evident that there may need to be a compromise when choosing between material attributes. For example, water uptake decreases due to higher density may reduce insulative properties as a result of fewer air gaps. Conversely, this huge range of growth outcomes imply we have only seen the tip of the iceberg as to what MBs are capable of and creates ample room for optimization.

When predicting production costs, these factors must also be taken into consideration as things like raw substrate used can influence this. Labour is however believed to be the largest contributing factor and is a prime candidate for autonomation. In addition, autonomous production processes could allow for increased scale and standardisation of production.

This technology could also open up novel manufacturing techniques and design tools. Integrating additive manufacturing, biomimetic processes and computerized topography optimization process into its internal structure also presents a unique opportunity to create ecological metamaterials with unsurpassed/dynamic insulation and structural properties. These could be used for not only insulation but a wide range of construction material or products to replace or even surpass conventional materials.

When comparing hydrocarbon insulators and their mycelium-based counterpart, we must not only consider their performance characteristics but also the environmental impacts throughout their whole lifecycle. Although exact numbers are not yet available in regards to mycelium, it is predicted that it holds a distinct advantage over the former at every stage from cradle to the grave. This is largely due to the use of widely available agricultural waste and the requirement for no energy input at the self-assembling growth stage of these composites. Furthermore, end of life biodegradability offers a unique opportunity to an additional raw material source.

\section{Conclusion}


Increasing attention has been paid to mycelium-based biocomposites as a green and economical alternative material to traditional EPS insulation panels. MBs have been shown to be competitive in terms of thermal, acoustic and fire safety although continual work is needed to address its higher water uptake and density. Furthermore, as composite substrates can utilize low cost readily available commercial waste products such as wheat husks, a clear value chain upscaling can be envisioned. MBs also hold a distinct advantage over conventional nonrenewable man-made materials due to their biodegradability and potential to be used as raw material at the end life. In addition, when comparing LCAs and production costs, MBs have significantly lower $\mathrm{CO} 2$ emissions and is believed to be much cheaper to produce. There is thus a clear opportunity to transform the current linear economy associated with insulation, towards a greener circular economy.

As MBs are grown rather than assembled, performance characteristics can vary widely depending on a number of factors and must be considered in its design conception and production. Furthermore, research into MBs is on going, making direct comparisons between studies more complicated.

Many of these issues presented could be potentially overcome through integrating emerging technologies such as 3D printing and topology optimisation into its production.

This could allow for refined control over internal structures and subsequent material performance. The combination of MB, 3D-printing of growth scaffolds and new computer support that combine topology optimization and knowledge of mycelium growth could enable designers to create a new generation of low weight, sustainable products utilizing renewable materials.

Aage, N., Andreassen, E., Lazarov, B. S., \& Sigmund, O. (2017). Giga-voxel computational morphogenesis for structural design. Nature, 550 (7674), 84-86. 
Abhijith, R., Ashok, A., \& Rejeesh, C. R. (2018). Sustainable packaging applications from mycelium to substitute polystyrene: a review. In Materials Today: Proceedings (Vol. 5, Issue 1, pp. 2139-2145).

Goidea, A., Floudas, D., \& Andreen, D. (2020). Pulp Faction: $3 d$ printed material assemblies through microbial biotransformation. 42-43.

Appels, F. V. W., Camere, S., Montalti, M., Karana, E., Jansen, K. M. B., Dijksterhuis, J., Krijgsheld, P., \& Wösten, H. A. B. (2019). Fabrication factors influencing mechanical, moisture- and water-related properties of mycelium-based composites. In Materials \& Design (Vol. 161, pp. 64-71).

Appels, F. V. W. (2020). The use of fungal mycelium for the production of bio-based materials (dr H A (ed.)) $[\mathrm{PhD}]$. Utrecht University.

Babrauskas, V., Levin, B. C., Gann, R. G., Paabo, M., Harris, R. H., Peacock, R. D., \& Yusa, S. (1992). Toxic potency measurement for fire hazard analysis. In Fire Technology (Vol. 28, Issue 2, pp. 163-167).

Bandyopadhyay, A., \& Chandra Basak, G. (2007). Studies on photocatalytic degradation of polystyrene. In Materials Science and Technology (Vol. 23, Issue 3, pp. 307-314).

Bell. (2017). Industrial Noise Control: Fundamentals and Applications, Second Edition. Routledge.

Boustead, I., Association of Plastics Manufacturers in Europe, \& European Centre for Plastics in the Environment. (1993). Eco-profiles of the European Plastics Industry: Polyethylene and polypropylene. Report 3.

Elsacker, E., Vandelook, S., Brancart, J., Peeters, E., \& De Laet, L. (2019). Mechanical, physical and chemical characterisation of mycelium-based composites with different types of lignocellulosic substrates. PloS One, 14(7), e0213954.

European Commission, editor. Being wise with waste: the EU's approach to waste management. (2010). Luxembourg: Publ. Off. of the European Union.

Fairs, M. Mycelium Chair by Eric Klarenbeek is 3D-printed with living fungus. Dezeen (2013). Available at: https://www.dezeen.com/2013/10/20/mycelium-chair-by-eric-klarenbeek-is-3d-printed-with-livingfungus/. (Accessed: 19th September 2019)

Grimm, D., \& Wösten, H. A. B. (2018). Mushroom cultivation in the circular economy. Applied Microbiology and Biotechnology, 102(18), 7795-7803.

Ismail, M. S., \& Waliuddin, A. M. (1996). Effect of rice husk ash on high strength concrete. In Construction and Building Materials (Vol. 10, Issue 7, pp. 521-526). https://doi.org/10.1016/0950-0618(96)00010-4

Jiang, L., Walczyk, D., McIntyre, G., \& Chan, W. K. (2016). Cost modeling and optimization of a manufacturing system for mycelium-based biocomposite parts. In Journal of Manufacturing Systems (Vol. 41, pp. 820).

Jones, M., Bhat, T., Huynh, T., Kandare, E., Yuen, R., Wang, C. H., \& John, S. (2018). Waste-derived low-cost mycelium composite construction materials with improved fire safety. In Fire and Materials (Vol. 42, Issue 7, pp. 816-825).

Jones, M., Bhat, T., Kandare, E., Thomas, A., Joseph, P., Dekiwadia, C., Yuen, R., John, S., Ma, J., \& Wang, C.H. (2018). Thermal Degradation and Fire Properties of Fungal Mycelium and Mycelium - Biomass Composite Materials. Scientific Reports, 8(1), 17583.

Jones, M., Mautner, A., Luenco, S., Bismarck, A., \& John, S. (2020). Engineered mycelium composite construction materials from fungal biorefineries: A critical review. In Materials \& Design (Vol. 187, p. 108397).

Kadoya, K., Matsunaga, N., \& Nagashima, A. (1985). Viscosity and Thermal Conductivity of Dry Air in the Gaseous Phase. In Journal of Physical and Chemical Reference Data (Vol. 14, Issue 4, pp. 947-970).

Lai, J. Walczyk, D. Mooney, L \& Putney, S. (2013). Manufacturing of mycelium-based biocomposites.

Leiva, F. J., Saenz-Díez, J. C., Martínez, E., Jiménez, E., \& Blanco, J. (2015). Environmental impact of Agaricus bisporus mycelium production. In Agricultural Systems (Vol. 138, pp. 38-45).

Liang, F. M., Liu, S. D., \& Chow, W. K. (2013). Study on Flashover in an Open Kitchen Fire by Nonlinear Dynamics. In Journal of Applied Fire Science (Vol. 23, Issue 2, pp. 157-178).

Lorente, S., \& Bejan, A. (2002). Combined 'flow and strength' geometric optimization: internal structure in a vertical insulating wall with air cavities and prescribed strength. In International Journal of Heat and Mass Transfer (Vol. 45, Issue 16, pp. 3313-3320).

Chandra, M. Kohn, C. Pawlitz, P \& Powell, G. (2016). REAL COST OF STYROFOAM. St. Louis Earth Day.

Miklečić, J., \& Jirouš-Rajković, V. (2016). Influence of Thermal Modification on Surface Properties and Chemical Composition of Beech Wood (Fagus sylvatica L.). In Drvna industrija (Vol. 67, Issue 1, pp. 65-71). https://doi.org/10.5552/drind.2016.1520

Nava, J. A. L., López Nava, J. A., Méndez González, J., Ruelas Chacón, X., \& Nájera Luna, J. A. (2016). Assessment of Edible Fungi and Films Bio-Based Material Simulating Expanded Polystyrene. In Materials and Manufacturing Processes (Vol. 31, Issue 8, pp. 1085-1090). https://doi.org/10.1080/10426914.2015.1070420 
Neviaser, J. L., \& Gann, R. G. (2004). Evaluation of Toxic Potency Values for Smoke from Products and Materials. In Fire Technology (Vol. 40, Issue 2, pp. 177-199).

Nissinen, A., Grönroos, J., Heiskanen, E., Honkanen, A., Katajajuuri, J.-M., Kurppa, S., Mäkinen, T., Mäenpää, I., Seppälä, J., Timonen, P., Usva, K., Virtanen, Y., \& Voutilainen, P. (2007). Developing benchmarks for consumer-oriented life cycle assessment-based environmental information on products, services and consumption patterns. In Journal of Cleaner Production (Vol. 15, Issue 6, pp. 538-549).

Oecd, OECD, \& Food and Agriculture Organization of the United Nations. (2009). Rice projections.

Papadopoulos, A. M. (2005). State of the art in thermal insulation materials and aims for future developments. In Energy and Buildings (Vol. 37, Issue 1, pp. 77-86).

Pelletier, M. G., Holt, G. A., Wanjura, J. D., Bayer, E., \& McIntyre, G. (2013). An evaluation study of mycelium based acoustic absorbers grown on agricultural by-product substrates. In Industrial Crops and Products (Vol. 51, pp. 480-485).

Rochman, C. M., Hoh, E., Hentschel, B. T., \& Kaye, S. (2013). Long-term field measurement of sorption of organic contaminants to five types of plastic pellets: implications for plastic marine debris. Environmental Science \& Technology, 47(3), 1646-1654.

Rossi, M., Camino, G., \& Luda, M. (2001). Characterisation of smoke in expanded polystyrene combustion. In Polymer Degradation and Stability (Vol. 74, Issue 3, pp. 507-512).

Sasson, A., \& Johnson, J. C. (2016). The 3D printing order: variability, supercenters and supply chain reconfigurations. In International Journal of Physical Distribution \& Logistics Management (Vol. 46, Issue 1, pp. 82-94).

Schiffman, R. (2013). Packing materials grown from mushrooms. In New Scientist (Vol. 218, Issue 2921, p. 29).

Shafiee, A., \& Atala, A. (2016). Printing Technologies for Medical Applications. In Trends in Molecular Medicine (Vol. 22, Issue 3, pp. 254-265).

Smunt, T. L., \& Meredith, J. (2000). A COMPARISON OF DIRECT COST SAVINGS BETWEEN FLEXIBLE AUTOMATION AND LABOR WITH LEARNING. In Production and Operations Management (Vol. 9, Issue 2, pp. 158-170).

Springer, G. S., \& Tsai, S. W. (1967). Thermal Conductivities of Unidirectional Materials. In Journal of Composite Materials (Vol. 1, Issue 2, pp. 166-173).

Stec, A. A., \& Richard Hull, T. (2011). Assessment of the fire toxicity of building insulation materials. In Energy and Buildings (Vol. 43, Issues 2-3, pp. 498-506).

The Voice of 3D Printing / Additive Manufacturing. (2015, February 2). 3DPrint.com | The Voice of 3D Printing / Additive Manufacturing. https://3dprint.com/41183/robotic-6-axis-3d-printer/ (Accessed: 20th September 2019)

Tabellini, G. Mycelium tectonics, studio di comportamenti morfologico-costruttivi attraverso sistemi biologici e simulazioni al comput. ( Università di Bologna, 2015).

Uysal, H., Demirboğa, R., Şahin, R., \& Gül, R. (2004). The effects of different cement dosages, slumps, and pumice aggregate ratios on the thermal conductivity and density of concrete. In Cement and Concrete Research (Vol. 34, Issue 5, pp. 845-848).

Website. (n.d.). Retrieved February 22, 2020, from Ecovative Design. Ecovative Design Available at: https://ecovativedesign.com

Website. (n.d.). Retrieved February 18, 2020, from Gryphon. Gryphon Available at: https://gryphon.newframeworks.com/.

Website. (n.d). Retrieved February 02, 2020, from Product Scorecard - Cradle to Cradle Products Innovation Institute. Available at:

https://www.c2ccertified.org/products/scorecard/mushroom_material

Xie, Z., Chen, L., \& Sun, F. (2014). Constructal optimization for an insulating wall combining heat flow, strength and volume. In International Communications in Heat and Mass Transfer (Vol. 54, pp. 141-147).

Xu, Q., \& Huang, Y. (2018). Anechoic and Reverberation Chambers. 
Yang, Z. (joey), Zhang, F., Still, B., White, M., \& Amstislavski, P. (2017). Physical and Mechanical Properties of Fungal Mycelium-Based Biofoam. In Journal of Materials in Civil Engineering (Vol. 29, Issue 7, p. 04017030).

Yu, C., Dai, C., \& Wang, B. J. (2007). Heat and mass transfer in wood composite panels during hot pressing: Part 3. Predicted variations and interactions of the pressing variables. In Holzforschung (Vol. 61, Issue 1, pp. 74-82 